\title{
Answer to the Letter to the Editor of M. Tyrakowski et al. concerning "Does whole-spine lateral radiograph with clavicle positioning reflect the correct cervical sagittal alignment?" by Park SM et al. (Eur Spine J; 24(1):57-62)
}

\author{
Kwang-Sup Song ${ }^{1} \cdot$ Hyun Kang ${ }^{1}$
}

Received: 10 April 2015/Revised: 10 April 2015/Accepted: 10 April 2015/Published online: 22 April 2015

(C) Springer-Verlag Berlin Heidelberg 2015

We would like to appreciate the comments on statistical concerns in our article entitled "Does whole-spine lateral radiograph with clavicle positioning reflect the correct cervical sagittal alignment?".

Selection of statistical methods or technique depends on the hypothesis and aim of the study in the planning phase. Researchers may pay more attention to the difference between measured variables or to the agreement between them.

A paired sample $t$ test is used to determine if there is a significant difference between the two variables, which are matched pairs of similar units, or one group of units measured twice. The null or alternative hypothesis of paired $t$ test is that the difference between two variables in the mean value is zero, or not. In contrary, intra-class correlation (one of reliability tests) is often used to assess the reliability of measurement or rating in continuous variables. The null or alternative hypothesis of intra-class correlation is that intra-class correlation coefficient is zero (or specific value) or not.

Among the concerns raised, first of all, our hypothesis was the arm-flexed clavicle position, which could affect cervical sagittal alignment as compared to the posture with arms extended and hands on either side. So, basically, the purpose of our study was focused to evaluate any

Kwang-Sup Song

ksong70@cau.ac.kr

1 Department of Orthopaedic Surgery, College of Medicine, Chung-Ang University, 224-1, Heukseok-dong, Dongjak-ku, Seoul 140-757, Republic of Korea

2 Anesthesiology, College of Medicine, Chung-Ang University, 224-1, Heukseok-dong, Dongjak-ku, Seoul 140-757, Republic of Korea differences of cervical sagittal alignments between two radiographic postures, standing cervical lateral radiograph and whole-spine lateral radiograph with clavicle positioning; it was not to test agreements between them and not to study for reporting reliability and agreement of specific parameters or measurement techniques. So, in our opinion, using paired $t$ test could be more appropriate.

In second concern, we agree with the comments, wherein intra-observer reproducibility with inter-observer reliability would be more valuable to demonstrate measurement accuracy. Interclass correlation coefficient values between two independent raters, shown in Table 1 in the article, were within a quite satisfactory ranges. So, the accuracy of measurement appeared to be adequate even though we didn't demonstrate intra-observer reproducibility.

Finally, two researchers evaluated all parameters in all enrolled patients as a matter of course. If they measured only on some sample of the whole subjects, we would have specified it on manuscript.

Overall, we totally agree that appropriate and clear methodological approach could promote the importance of conclusions of reported studies and appreciate the opportunity to review the above issues.

Conflict of interest None. 